Published in final edited form as:

Am J Geriatr Psychiatry. 2004 ; 12(2): 179-188.

\title{
Familial Occurrence of Dementia With Lewy Bodies
}

Debby W. Tsuang, M.D., Lillian DiGiacomo, B.A., and Thomas D. Bird, M.D. From the Departments of Psychiatry and Behavioral Sciences (DWT,TDB), Neurology (TDB), and Medical Genetics (TDB), University of Washington, Seattle, WA; the Mental IIIness Research,Education, and Clinical Center (DWT,LD), and the Geriatric Research, Education, and Clinical Center (TDB), VA Puget Sound Health Care System, Seattle, WA.

\section{Abstract}

Objective. The authors investigated the validity of the designation "familial dementia with Lewy bodies (DLB)" by evaluating the clinical, neuropathological, and genetic characteristics of previously reported families exhibiting both familial parkinsonism and dementia.

Methods. Several families, including multiple individuals with parkinsonism as well as prominent dementia, were identified through a literature search. Selected families had at least one member with dementia with autopsy evidence of neocortical and/or limbic Lewy-body (LB) pathology. Clinical and neuropathological evidence from reports of families with prominent dementia as well as parkinsonism was reviewed to further define familial DLB.

Results. All selected families had at least one affected individual with dementia and autopsy-proven DLB. Therefore, these families might be considered examples of familial DLB. Individuals in the first six families typically presented with parkinsonian features, whereas cognitive decline did not appear until years later. In contrast, in the other six families, affected individuals typically presented with cognitive decline, and parkinsonism developed later.

Conclusions. Families exist in which one or more persons meet both clinical and neuropathological criteria for DLB. They differ as to whether the signs of parkinsonism precede or follow signs of dementia. It remains to be determined whether this clinical distinction is biologically important. Susceptibility to developing LB pathology may be determined by the interaction between genetic predisposition and environmental risk factors.

Dementia with Lewy bodies (DLB) accounts for what may be the second-largest neuropathological subgroup of dementing illnesses, preceded only by Alzheimer disease (AD). ${ }^{1}$ Clinical characteristics of DLB include cognitive decline, parkinsonian signs and symptoms, fluctuations in cognition and attention, and visual hallucinations. ${ }^{2}$ Substantial clinical overlap exists between DLB and AD, as well as between DLB and Parkinson disease (PD). This has resulted in frequent misdiagnosis of DLB. Clinically, patients with DLB generally exhibit hallucinations earlier in the course of illness than patients with AD. The motor signs and symptoms of PD may also be observed in patients with DLB, though such symptoms are often less severe than in patients with PD. Given previous neuropathological studies suggesting that DLB may be a distinct diagnostic entity, 3,4 the ability to distinguish DLB from AD and PD is important.

Consensus guidelines for the clinical diagnosis of DLB were established by the Consortium on Dementia with Lewy Bodies in 1996. ${ }^{2}$ The primary criterion for the clinical diagnosis of DLB is progressive cognitive impairment of sufficient severity to disrupt normal functioning. A decline in memory, visuospatial, and frontal-subcortical skills may also be noted. ${ }^{5}$ Other

Send correspondence to Debby Tsuang, M.D., M.Sc., VAPSHCS (116-MIRECC), 1660 South Columbian Way, Seattle, WA 98108. email: dwt1@u.washington.edu. 
central features of DLB include the following: 1) fluctuating cognition (rapid or slow), with prominent changes in attention and awareness early in the course of illness; ${ }^{6}$ ) complex and recurring visual hallucinations (often relating to animals or children ${ }^{7}$ ); and 3 ) spontaneous parkinsonian features. In addition to the primary criterion, two of these three features are required for a diagnosis of probable DLB, whereas one is necessary for a diagnosis of possible DLB. Furthermore, parkinsonism, if present, should not precede the onset of dementia by more than 1 year.

Although the sensitivity (correctly diagnosing DLB) of these diagnostic criteria is modest, the specificity (correctly not diagnosing DLB) is high. This finding most likely results from the clinical variability seen in DLB and is reflected in its diagnostic criteria: although all affected patients exhibit cognitive impairment, the presence of the other core features is much less consistent. 8,9

DLB is typically considered to be a sporadic disorder. However, given that genetic factors have been identified in PD, ${ }^{10}$ it is possible that genetic factors may also play a role in DLB. Indeed, kindreds with familial DLB have been reported. ${ }^{11}$ One approach to investigating the clinical and neuropathological variability within DLB is to identify and evaluate kindreds with familial DLB, thereby enabling further study of the aggregation of core clinical symptoms within families. Studies in these families can provide important insights into the clinical and pathophysiological features of this increasingly recognized disorder.

Numerous reports have identified kindreds with inherited parkinsonism and dementia. 12 Dementia commonly develops in patients with parkinsonism. The majority of these reports describe cases in which parkinsonism preceded the onset of dementia by many years, and are therefore diagnosed as cases of PD with dementia. In contrast, few families in which dementia precedes the onset of parkinsonism have been described. ${ }^{11,13}$ Moreover, limited neuropathological data have been available to evaluate those patients who first present with dementia and then later develop parkinsonism. Recent neuropathological data for this small number of families suggests that they may represent cases of familial DLB.

To investigate whether familial DLB exists, we identified several families in the literature that include multiple individuals with parkinsonism as well as prominent dementia. Selected families also had at least one autopsy demonstrating neocortical Lewy-body (LB) pathology (with one exception). In this study, we use clinical and neuropathological evidence from previous reports of families with prominent dementia within the context of parkinsonism to further define DLB.

\section{METHODS}

To find studies on kindreds with DLB, we used the PubMed database

(www.ncbi.nlm.nih.gov). We identified approximately 570 indexed articles addressing familial parkinsonism. As expected, when we reviewed the selected articles, the majority of affected members in these families had predominant parkinsonism. $12,14,15$ If dementia was present in these families, it occurred in very few affected individuals. ${ }^{12}$ Furthermore, many of the studies included only autopsy-confirmed cases with substantia-nigral LBs. These studies were excluded. We identified 12 extended families that met our search criteria of familial parkinsonism with prominent dementia, as well as autopsy-confirmed neocortical and/or limbic LB pathology in at least one family member with dementia. As will be seen, in some families, parkinsonism preceded the onset of dementia. These families are included here because their dementia was a prominent feature of the phenotype, and LBs were found outside the substantia nigra. 
We reviewed the descriptions of these families and extracted composite clinical, genetic, and neuropathological information from the publications. Whenever possible, descriptions of families reported in multiple publications were combined to avoid duplication.

\section{RESULTS}

See Table 1 for composite information on families. Family data are presented in chronological order by publication date.

\section{Contursi Kindred}

Golbe and Duda describe an extensive family with parkinsonism from the small town of Contursi, in the province of Salerno, Italy, who carry the A53T alpha-synuclein mutation. ${ }^{16-}$ 18 In this family, 60 affected individuals, 14 of whom have been evaluated clinically, have been identified across 6 generations. Severity of dementia varied widely across affected members of this family, with onset typically following the onset of parkinsonism (the precise onset of dementia was often unclear). In the few individuals who developed psychosis, hallucinations and delusions were the most common symptoms.

Autopsies were obtained on two individuals from this family. ${ }^{16}$ Classic LBs were exceedingly rare. However, alpha-synuclein immunostaining was not performed. Neither individual demonstrated any evidence of AD pathology.

\section{lowa Kindred}

Waters and Miller, ${ }^{19}$ Muenter et al., ${ }^{20}$ and Farrer et al., ${ }^{21}$ provided clinical, genetic, and neuropathological information on a family with 22 affected individuals across six generations. Ultimately, 12 of these individuals were clinically examined. Specific parkinsonian features included postural instability, cogwheel rigidity, masked face, rest tremor, dysarthria, and gait changes. Four individuals initially responded well to levodopa therapy. Dementia consisted primarily of memory impairment, visuospatial dysfunction, and disorientation. In one individual, visual hallucinations improved after discontinuation of levodopa therapy, but did not completely resolve. The mode of inheritance in this family is most consistent with autosomal dominant transmission. Although parkinsonism tends to occur first in this family, we include them in our review because of the frequency and prominence of later dementia.

Six autopsies were performed. Microscopic examination revealed LB pathology and moderateto-severe cell loss in the substantia nigra in all six affected individuals. In the first case, cortical LBs were fairly numerous in all limbic areas (i.e., parahippocampal gyrus and amygdala). Occasional LBs were found in the deep cortex. Substantia-nigral LBs stained with ubiquitin were atypical in this case, without the eosinophilic central core, concentric lamellae, or clear peripheral halo of LBs seen in idiopathic PD. Four other cases had almost identical gross neuropathological findings, exhibiting widespread occurrence of subcortical and cortical LBs. 20 In these four cases, LBs were numerous and of unusual shape. The final autopsy case, an individual with dementia, parkinsonism, and psychosis, demonstrated neuropathology also relatively similar to the first five. Microscopically, alpha-synuclein immunostaining demonstrated unusual round-to-pleomorphic intraneuronal inclusions in the lower cortical layers, consistent with cortical LBs. This case was determined to represent the neocortical stage of DLB. None of the six cases met neuropathological criteria for AD.

Genome-wide linkage analysis in this extended family demonstrated that an inferred haplotype on Chromosome 4p (D4S1609-D4S2408) segregated with the disease. ${ }^{21}$ However, individuals with postural tremors without other parkinsonian features also shared this haplotype. Maximum multipoint linkage analysis using only affected individuals gave a 
maximum z-score of 2.64 at D4S1609, designated as PARK4. Recently, Singleton and colleagues reported the presence of an alpha-synuclein whole gene triplication in the affected individuals of this family. ${ }^{22}$ An overexpression of normal alpha-synuclein gene was observed in these individuals. Identification of this new genetic mutation further solidifies the causative role of alpha-synuclein in PD.

\section{Denson: Family G}

Denson $^{23}$ reports on Family G, a family with 10 affected individuals, 4 of whom were examined clinically, across 3 generations. Four individuals with parkinsonism-only were responsive to levodopa therapy. In the three individuals presenting with cognitive disturbance, dementia consisted primarily of memory impairment.

One autopsy was performed on a family member who presented with parkinsonism at age 48 and developed dementia at age 70. Gross neuropathological findings demonstrated mild cortical atrophy and a severely depigmented substantia nigra. Microscopic examinations revealed ubiquitin-positive LBs in the substantia nigra, locus ceruleus, and nucleus basalis of Meynert. LBs were also present in the neocortex, amygdala, hypothalamus, and dorsal vagal nucleus. These findings are consistent with the neocortical form of DLB. There were rare, scattered, and indistinct neuritic plaques and neurofibrillary tangles, not sufficient to meet criteria for $\mathrm{AD}$.

The transmission pattern in Family $\mathrm{G}$ is consistent with autosomal-dominant inheritance, and evidence for linkage to Chromosome 2p13 has been reported and designated as PARK3. ${ }^{24}$ The maximum z-score in Family $\mathrm{G}$ is 1.13 for 2 p13 markers.

\section{Ishikawa: Family A}

Ishikawa et al. ${ }^{25}$ and Wakabayashi et al. ${ }^{26}$ described the clinical and neuropathological characteristics, respectively, of Family A, a kindred with four affected individuals throughout three generations, all of whom were evaluated clinically by Ishikawa and colleagues. ${ }^{25,26}$ All four affected individuals presented with levodopa-responsive parkinsonism, followed by dementia and psychosis. Parkinsonian signs and symptoms in this family included gait disturbance, hand tremor, masked face, bradykinesia, cogwheel rigidity, and absence of postural reflex; however, rigidity and tremor were absent in one individual. Dementia characteristics were generally limited to memory disturbance. Behavioral symptoms included delusions and visual hallucinations in three individuals and bizarre behavior in two individuals.

Two autopsies were performed. Neuropathological evaluation in both individuals revealed significant depigmentation of the substantia nigra and locus ceruleus. Except for slight frontal lobe atrophy, there were no other gross abnormalities. Ubiquitin-positive LBs were present in the substantia nigra and the deep layer of the cerebral cortex. Senile plaque and neurofibrillary tangle findings were exceedingly mild and insufficient to meet neuropathological criteria for AD. Both individuals fulfilled criteria for the neocortical form of DLB.

The inheritance pattern in Family A is consistent with autosomal-dominant transmission despite consanguinity (the proband married her uncle). Three affected individuals carried the $\varepsilon 3 / \varepsilon 4$ genotype, and one affected individual carried the $\varepsilon 4 / \varepsilon 4$ genotype.

\section{Ohara: Familial Dementia With Lewy Bodies}

Ohara et al. ${ }^{27}$ describe a family with three affected individuals in one generation, all of whom were clinically examined. ${ }^{27}$ All three exhibited progressive dementia with early onset, as well as psychosis, and two also had parkinsonian features. Parkinsonian signs and symptoms primarily consisted of clumsiness, gait disturbance, rigidity, masked face, and bradykinesia. 
Treatment of parkinsonism with levodopa therapy was effective in one individual, but relatively ineffective in the other. Dementia features included memory disturbance, dysarthria, word-finding difficulty, and paraphasia. Psychiatric features, noted during the period of increasing cognitive decline, included delusions of persecution (in all individuals), sexually uninhibited behaviors (in two individuals), visual hallucinations (in two individuals), and compulsive behavior (in one individual).

An autopsy was performed on an individual with dementia, parkinsonism, and psychosis. Neuropathological findings revealed depigmentation in the substantia nigra. Microscopic examination revealed numerous ubiquitin-positive LBs in the brainstem and throughout the cerebral cortex. Neurofibrillary tangles and senile plaques were not detected.

The pattern of inheritance in this family is most consistent with autosomal-recessive inheritance. DNA from one affected individual was screened for known mutations in the alphasynuclein gene (A53T and A30P) and ubiquitin carboxy-terminal hydrolase L1 gene, as well as deletions in the parkin gene. No mutations were found.

\section{Spira: Australian Family of Greek Origin}

Spira et al. ${ }^{28}$ describe an Australian family of Greek origin with the A53T alpha-synuclein mutation. In this family, there are five affected individuals in one generation, and three of them were examined clinically. All five demonstrated dementia and parkinsonism, and two also exhibited psychotic features. Parkinsonian features in this family included decreased arm swing, bradykinesia, rigidity, and frequent falls. The three individuals who were examined clinically all had a positive response to levodopa therapy. Dementia consisted primarily of cognitive slowing, short attention span, and confusion. Psychiatric characteristics in this family included acute paranoia in both psychotic individuals, and hallucinations in only one of them.

Two individuals, one with psychosis and one without, underwent postmortem examination. Macroscopically, both exhibited marked depigmentation of the substantia nigra and locus ceruleus. LBs were detected in many of the remaining neurons of these regions. Cortical LBs were detected in the cortex of the individual without psychosis, but they were not observed in the psychotic individual. No AD pathology was present in either case.

\section{Brett: Family 1}

Brett et al. ${ }^{29}$ describe two affected siblings, both of whom underwent a clinical evaluation. Dementia, parkinsonism, and psychosis were present in both individuals. Precise ages at onset for parkinsonism and dementia were not available. Dementia features included memory impairment and progressive decline in one sibling, but not the other. Parkinsonian signs included impaired balance, gait changes, rigidity, bilateral cogwheeling, and bradykinesia. Psychosis consisted of visual hallucinations in both individuals.

Autopsies were performed in both siblings. Macroscopic findings in both individuals revealed pallor in the substantia nigra. Microscopic findings included LBs in the substantia nigra and locus ceruleus of both patients. Cell loss, gliosis, and depigmentation were also identified in both of these locations. Although occasional tau-positive neurofibrillary tangles were seen in the hippocampus of one individual, neither individual had sufficient pathology to fulfill criteria for a diagnosis of $\mathrm{AD}$.

\section{Galvin: Family 1 and Family 2}

Galvin et al. report on two families. ${ }^{30}$ Family 1 includes two affected individuals in two generations, both of whom were evaluated clinically. Dementia, parkinsonism, and psychosis were determined to be present in both individuals. Whereas one individual presented with 
parkinsonism, the other individual presented with dementia. Parkinsonian features included gradual onset of "stiffness" and rigidity in one individual, with postural instability and gait impairment in the other individual. Dementia consisted primarily of forgetfulness, as well as impaired psycho-motor performance, while psychosis included visual hallucinations in both individuals, as well as delusions in one individual.

Both family members underwent autopsy. In one case, there was marked depigmentation of the substantia nigra. In the other case, the substantia nigra was normal. In both cases, alphasynuclein-positive LBs were present in the substantia nigra and neocortex, whereas senile plaques and neurofibrillary tangles were generally absent.

Family 2 also includes two affected individuals in two generations, both of whom were clinically examined. Although both were found to have parkinsonism and dementia, only one also displayed psychotic symptoms. Parkinsonian signs and symptoms included cogwheel rigidity, bradykinesia, and abnormal gait. Dementia consisted mainly of progressive memory decline, disorientation, aphasia, and personality change. One individual demonstrated ongoing paranoid delusions.

Both individuals underwent autopsy. In the individual with psychosis, there was mild depigmentation of the substantia nigra; however, in the other case, the substantia nigra was normal. In both cases, microscopic examination revealed the presence of alpha-synucleinpositive LBs throughout the neocortex, hippocampus, and substantia nigra. Senile plaques and neurofibrillary tangles were similarly numerous in both cases, sufficient to fulfill neuropathological criteria for AD.

The pattern of inheritance in Family 2 is consistent with autosomal-dominant transmission. No pathogenic mutations or polymorphisms in the alpha-synuclein gene were found in either family.

\section{Tsuang: Family 1 and Family 2}

We have previously described two unrelated families. ${ }^{11}$ Family 1 consists of eight affected individuals across two generations. One of the affected individuals was clinically evaluated. Five affected individuals were determined to have parkinsonism; four were determined to have psychosis; and all eight exhibited dementia as the presenting symptom. Parkinsonian signs and symptoms in this family included cogwheel rigidity, stooped posture, and bradykinesia. Dementia symptoms included forgetfulness, word-finding difficulty, impaired judgment, poor executive function, and behavioral changes. Psychotic symptoms included visual hallucinations in two individuals, and paranoid ideation in two other individuals.

Three autopsies were performed. All autopsied individuals had dementia, parkinsonism, and psychosis. Microscopic findings in one individual demonstrated sufficient senile plaque and neurofibrillary tangle pathology (Braak stage $\mathrm{V}-\mathrm{C}$ ) to meet neuropathological criteria for AD. In this same case, alpha-synuclein-positive LBs were detected in the amygdala only. Therefore, this individual may represent a case of amygdala-predominant DLB, a subtype that has been described elsewhere ${ }^{31}$ and the significance of which remains unclear. The other two individuals had no significant gross findings, and the substantia nigra appeared to be wellpigmented. However, on microscopic examination, these individuals exhibited LBs in the substantia nigra and the neocortex, with only mild AD pathology (both, Braak stage III). ${ }^{32}$

Family 2 includes six affected individuals in one generation, four of whom were examined clinically. All six individuals presented with dementia, and four also demonstrated parkinsonism, either concurrently or later in the course of illness. Cognitive decline in this family included memory impairment, word-finding difficulty, disorientation, poor executive 
function, and apraxia. Parkinsonian features in this family included stooped posture, bradykinesia, gait instability, and masked face.

Autopsies were performed in two out of six individuals with dementia. There was no depigmentation of the substantia nigra. One individual demonstrated sufficient senile plaque and neurofibrillary tangle pathology (Braak stage VI-C) to meet neuropathological criteria for AD. ${ }^{32}$ In this same case, LBs were detected in the amygdala only, again perhaps representing a case of amygdala-predominant DLB. The other individual exhibited LBs in the substantia nigra and the neocortex, with only mild AD pathology (Braak stage III).

Family 1 exhibits a familial pattern consistent with autosomal-dominant transmission. Seven individuals in Family 1 (six affected and one at-risk) and four individuals in Family 2 (all affected) had DNA available. In Family 1, five affected individuals carried the APOE $\varepsilon 3 / \varepsilon 4$ genotype, whereas the other affected individual had an APOE $\varepsilon 4 / \varepsilon 4$ genotype. The at-risk individual also had an APOE $\varepsilon 3 / \varepsilon 4$ genotype. In Family 2, three affected individuals had the APOE $\varepsilon 4 / \varepsilon 4$ genotype, whereas one affected individual had the APOE $\varepsilon 3 / \varepsilon 4$ genotype. Genetic analyses showed no nucleotide alterations in the alpha-, beta-, gamma-synuclein, or parkin genes in one affected individual selected from each family. There were no deletions or duplications in the parkin gene in the individuals examined.

\section{Bonner: Familial Dementia With Lewy Bodies}

Bonner et al. ${ }^{13}$ report on a family with eight affected individuals (one of whom was clinically evaluated) spanning two generations. All eight individuals presented with cognitive decline; four individuals later demonstrated parkinsonian features, and four later developed psychosis. Dementia symptoms included memory and language disturbance, word-finding difficulty, executive dysfunction, disorientation, and visual-spatial dysfunction. Parkinsonian features of this family included bradykinesia, tremor, cogwheel rigidity, as well as gait and postural disturbances. Psychotic symptoms included behavioral disturbances, such as auditory and visual hallucinations, agitation, paranoia, verbal outbursts, and other bizarre behaviors.

One autopsy was performed in an individual with dementia, parkinsonism, and hallucinations. On gross examination, findings were characterized by substantia nigral depigmentation.

Microscopic examination revealed widespread brainstem and cortical alpha-synuclein-positive LBs, specifically in the substantia nigra, cingulate gyrus, parahippocampal gyrus, and amygdala. Senile plaque formation in the cortex and hippocampus, as well as neurofibrillary tangles in the medial temporal lobe and hippocampus indicated moderate-to-severe AD pathology (Braak stage V). As such, this case fulfilled neuropathological criteria for DLB (neocortical subtype) and AD.

This family demonstrates an autosomal-dominant pattern of transmission. The proband's APOE genotype was $\varepsilon 3 / \varepsilon 4$. There were no alternations in the alpha- or gamma-synuclein genes. However, there was a nucleotide change in a highly conserved region of the beta-synuclein gene. This nucleotide substitution results in an amino acid change from proline to histidine. This nucleotide change was not detected in 415 control individuals. ${ }^{33}$ Although the family included seven other affected individuals (all but one deceased), ${ }^{13}$ DNA is unavailable from any other autopsied relatives to confirm that this is a disease-causing mutation.

\section{CONCLUSIONS}

The present study describes the clinical, neuropathological, and genetic characteristics of 12 families exhibiting familial parkinsonism and dementia. All families had at least one affected individual with dementia and autopsy-proven DLB. Therefore, these families might be considered examples of familial DLB. Individuals in the first six families described here 
typically presented with parkinsonian features, with cognitive decline not appearing until years later. In contrast, in the other six families, affected individuals typically presented with cognitive decline, while parkinsonism developed later. Individuals in the first six families would not meet consensus criteria for $\mathrm{DLB}^{2}$ because the onset of parkinsonism preceded the onset of dementia by more than 1 year. In two of these families, affected individuals carry the A53T alpha-synuclein mutation. In another family, affected individuals carry a newlyidentified mutation that is characterized as a triplication of the alpha-synuclein gene. Meanwhile, in one other family, there was evidence for linkage to PARK3. Therefore, these families might be designated as kindreds with familial parkinsonism-alone. It remains unclear whether this distinction (i.e., the difference in presenting symptoms) has pathophysiological significance.

It has been suggested that LB disease includes a spectrum of clinical-pathological entities ranging from $\mathrm{PD}$ to the $\mathrm{LB}$ variant of $\mathrm{AD}$. Others have previously suggested that concomitant AD pathology in PD cases was associated with cognitive decline. ${ }^{34,35}$ In the families that we reviewed, none of the cases that presented with parkinsonism had sufficient AD pathology to meet criteria for $\mathrm{AD}$. On the other hand, cases that presented with early cognitive decline almost always demonstrated concomitant $\mathrm{AD}$ pathology sufficient to meet criteria for $\mathrm{AD} .11,13,30$

Alternatively, cognitive decline in these families may be associated with the density of cortical LBs. ${ }^{36-38}$ However, in the families presented here, LB density was not evaluated, nor did all cases undergo alpha-synuclein immunostaining. Therefore, direct assessment of this relationship is not possible. Our observations are also limited by the availability of only one or two autopsies in the majority of these families. Furthermore, although the availability of alpha-synuclein-specific antibodies has provided a new tool for the identification of LBs in brain tissue, not all of these studies conducted alpha-synuclein immunostaining or extra-nigral sampling for the detection of LBs. Therefore, autopsy findings may not be directly comparable between studies. Furthermore, not all cases described had typical LB pathology associated with PD. ${ }^{19}$ Such families may represent a separate disease entity. Future neuropathological studies using up-to-date immunostaining in multiple affected individuals from the same family are necessary.

Many genetic studies have suggested that familial PD and familial AD may share certain genetic factors. Using age-at-onset data, $\mathrm{Li}$ and colleagues, ${ }^{40}$ conducted a linkage analysis of families having either AD or PD. Initial genomic screens revealed that although age at onset in $\mathrm{AD}$ and $\mathrm{PD}$ have evidence for linkage to separate chromosomal regions, there is also evidence for linkage to Chromosomes 6 and 10 in both disorders. Although AD and PD are thought to be distinct clinical and pathological entities, they likely share some genetic risk factors. However, the relationship of familial syndromes to sporadic, idiopathic AD or PD remains unclear.

Furthermore, genetic-linkage studies suggest that the LB variant of AD may be a distinct genetic subtype. Scott and colleagues ${ }^{39}$ reported evidence for linkage to markers D12S398 and D12S1632 in families with multiple affected individuals with late-onset AD. The evidence for linkage was strongest in families with affected individuals lacking an APOE $\varepsilon 4$ allele, as well as in families in which there was at least one affected individual with a neuropathological diagnosis of DLB. However, most of these families included only one individual who had undergone autopsy. Again, additional genetic studies in extended families providing multiple autopsies are necessary in order to investigate whether DLB segregates within families.

In summary, families exist in which one or more persons meet both clinical and neuropathological criteria for DLB. They differ as to whether the signs of parkinsonism precede or follow the signs of dementia. It remains to be determined whether this clinical distinction 
is biologically important. Substantial clinical and genetic heterogeneity exists within DLB. Identification of susceptibility genes in familial DLB may shed light on the clinical and pathological overlap between AD and PD. Both disorders show abnormal protein deposits that develop before neuronal degeneration. Identification of novel gene products may result in the detection of additional pathology in DLB. Further investigations, including detailed clinical and neuropathological descriptions of multiple affected individuals in these families, are necessary to increase our knowledge of this disorder.

\section{References}

1. Barber R, Panikkar A, McKeith IG. Dementia with Lewy bodies: diagnosis and management. Int J Geriatr Psychiatry 2001;16:S12-S18. [PubMed: 11748785]

2. McKeith I, Galasko D, Kosaka K, et al. Consensus guidelines for the clinical and pathologic diagnosis of dementia with Lewy bodies (DLB): Report of the Consortium on DLB International Workshop. Neurology 1996;47:1113-1124. [PubMed: 8909416]

3. Hansen L, Salmon D, Galasko D, et al. The Lewy-body variant of Alzheimer's disease: a clinical and pathologic entity. Neurology 1990;40:1-8. [PubMed: 2153271]

4. Kosaka K. Diffuse Lewy-body disease in Japan. J Neurol 1990;237:197-204. [PubMed: 2196340]

5. Salmon D, Galasko D: Neuropsychological aspects of Lewy-body dementia, in Dementia With Lewy Bodies. Edited by Perry R, McKeith I, Perry E. Cambridge, UK, Cambridge University Press, 1996, p 99-133

6. Walker MP, Ayre GA, Cummings JL, et al. Quantifying fluctuation in dementia with Lewy bodies, Alzheimer's disease, and vascular dementia. Neurology 2000;54:1616-1625. [PubMed: 10762503]

7. Ballard C, McKeith I, Harrison R, et al. A detailed phenomenological comparison of complex visual hallucinations in dementia with Lewy bodies and Alzheimer's disease. Int Psychogeriatr 1997;9:381388. [PubMed: 9549588]

8. Lopez O, Becker J, Kaufer D, et al. Research evaluation and prospective diagnosis of dementia with Lewy bodies. Arch Neurol 2002;59:43-46. [PubMed: 11790229]

9. Holmes C, Cairns N, Lantos P, et al. Validity of current clinical criteria for Alzheimer's disease, vascular dementia, and dementia with Lewy bodies. Br J Psychiatry 1999;174:45-50. [PubMed: 10211150]

10. Polymeropoulos M, Higgins J, Golbe L, et al. Mapping of a gene for Parkinson's disease to Chromosome 4q21-q23. Science 1996;274:1197-1199. [PubMed: 8895469]

11. Tsuang DW, Dalan AM, Eugenio CJ, et al. Familial dementia with Lewy bodies: a clinical and neuropathological study of two families. Arch Neurol 2002;59:1622-1630. [PubMed: 12374501]

12. Denson M, Wszolek Z. Familial parkinsonism: our experience and review. Parkinsonism and Related Disorders 1995;1:35-46.

13. Bonner L, Tsuang D, Cherrier M, et al. Familial dementia with Lewy bodies with an atypical clinical presentation. J Geriatr Psychiatry Neurol 2003;16:59-64. [PubMed: 12641375]

14. Papapetropoulos S, Paschalis C, Athanassiadou A, et al. Clinical phenotype in patients with alphasynuclein Parkinson's disease living in Greece in comparison with patients with sporadic Parkinson's disease. J Neurol Neurosurg Psychiatry 2001;70:662-665. [PubMed: 11309462]

15. Rosenberg R, Green J, White C, et al. Dominantly inherited dementia and parkinsonism, with nonAlzheimer amyloid plaques: a new neurogenetic disorder. Ann Neurol 1989;25:152-158. [PubMed: 2645825]

16. Golbe L, Di Iorio G, Bonavita V, et al. A large kindred with autosomal dominant Parkinson's disease. Ann Neurol 1990;27:276-282. [PubMed: 2158268]

17. Golbe LI, Di Iorio G, Sanges G, et al. Clinical genetic analysis of Parkinson's disease in the Contursi kindred. Ann Neurol 1996;40:767-775. [PubMed: 8957018]

18. Duda JE, Giasson BI, Mabon ME, et al. Concurrence of alpha-synuclein and tau brain pathology in the Contursi kindred. Acta Neuropathol (Berl) 2002;104:7-11. [PubMed: 12070658]

19. Waters C, Miller C. Autosomal dominant Lewy-body parkinsonism in a four-generation family. Ann Neurol 1994;35:59-64. [PubMed: 8285594] 
20. Muenter MD, Forno LS, Hornykiewicz O, et al. Hereditary form of parkinsonism-dementia. Ann Neurol 1998;43:768-781. [PubMed: 9629847]

21. Farrer M, Gwinn-Hardy K, Muenter M, et al. A chromosome $4 p$ haplotype segregating with Parkinson's disease and postural tremor. Hum Mol Genet 1999;8:81-85. [PubMed: 9887334]

22. Singleton AB, Farrer M, Johnson J, et al. Alpha-synuclein locus triplication causes Parkinson's disease. Science 2003;302:841. [PubMed: 14593171]

23. Denson M, Wszolek Z, Pfeiffer R, et al. Familial parkinsonism, dementia, and Lewy-body disease: study of Family G. Ann Neurol 1997;42:638-643. [PubMed: 9382476]

24. Gasser T, Muller-Myhsok B, Wszolek ZK, et al. A susceptibility locus for Parkinson's disease maps to Chromosome 2p13. Nat Genet 1998;18:262-265. [PubMed: 9500549]

25. Ishikawa A, Takahashi $\mathrm{H}$, Tanaka $\mathrm{H}$, et al. Clinical features of familial diffuse Lewy body disease. Eur Neurol 1997;38:34-38. [PubMed: 9276199]

26. Wakabayashi K, Hayashi S, Ishikawa A, et al. Autosomal dominant diffuse Lewy-body disease. Acta Neuropathol 1998;96:207-210. [PubMed: 9705138]

27. Ohara K, Takauchi S, Kokai M, et al. Familial dementia with Lewy bodies (DLB). Clin Neuropathol 1999;18:232-239. [PubMed: 10505432]

28. Spira PJ, Sharpe DM, Halliday G, et al. Clinical and pathological features of a parkinsonian syndrome in a family with an Ala53Thr alpha-synuclein mutation. Ann Neurol 2001;49:313-319. [PubMed: 11261505]

29. Brett FM, Henson C, Staunton H. Familial diffuse Lewy-body disease, eye movement abnormalities, and distribution of pathology. Arch Neurol 2002;59:464-467. [PubMed: 11890854]

30. Galvin JE, Lee SL, Perry A, et al. Familial dementia with Lewy bodies: clinicopathologic analysis of two kindreds. Neurology 2002;59:1079-1082. [PubMed: 12370468]

31. Dickson D, Corral A, Lin W, et al. Alzheimer's disease with amygdaloid Lewy bodies: a form of Lewy-body disease distinct from Alzheimer's disease and diffuse Lewy-body disease (abstract). Neurology 2000;54:A451.

32. National Institute on Aging. Consensus recommendations for the postmortem diagnosis of Alzheimer's disease: The National Institute on Aging, and Reagan Institute Working Group on Diagnostic Criteria for the Neuropathological Assessment of Alzheimer's Disease. Neurobiol Aging 1997;18:S1-S2. [PubMed: 9330978]

33. Limprasert $P$, Taylor J, Leverenz J, et al. Beta-synuclein gene alteration in dementia with Lewy bodies (abstract). Amer J Hum Genet 2001;69:196.

34. Hughes A, Daniel S, Blankson S, et al. A clinicopathological study of 100 cases of Parkinson's disease. Arch Neurol 1993;50:140-148. [PubMed: 8431132]

35. Jendroska K. The relationship of Alzheimer-type pathology to dementia in Parkinson's disease. J Neural Transm Suppl 1997;49:23-31. [PubMed: 9266411]

36. Hurtig HI, Trojanowski JQ, Galvin J, et al. Alpha-synuclein cortical Lewy bodies correlate with dementia in Parkinson's disease. Neurology 2000;54:1916-1921. [PubMed: 10822429]

37. Richard IH, Papka M, Rubio A, et al. Parkinson's disease and dementia with Lewy bodies: one disease or two? Mov Disord 2002;17:1161-1165. [PubMed: 12465052]

38. Samuel W, Galasko D, Masliah E, et al. Neocortical Lewy-body counts correlate with dementia in the Lewy-body variant of Alzheimer's disease. J Neuropathol Exp Neurol 1996;55:44-52. [PubMed: 8558171]

39. Scott WK, Grubber JM, Conneally PM, et al. Fine mapping of the Chromosome-12 late-onset Alzheimer disease locus: potential genetic and phenotypic heterogeneity. Am J Hum Genet 2000;66:922-932. [PubMed: 10712207]

40. Li YJ, Scott WK, Hedges DJ, et al. Age at onset in two common neurodegenerative diseases is genetically controlled. Am J Hum Genet 2002;70:985-993. [PubMed: 11875758] 
Tsuang et al.

Page 11

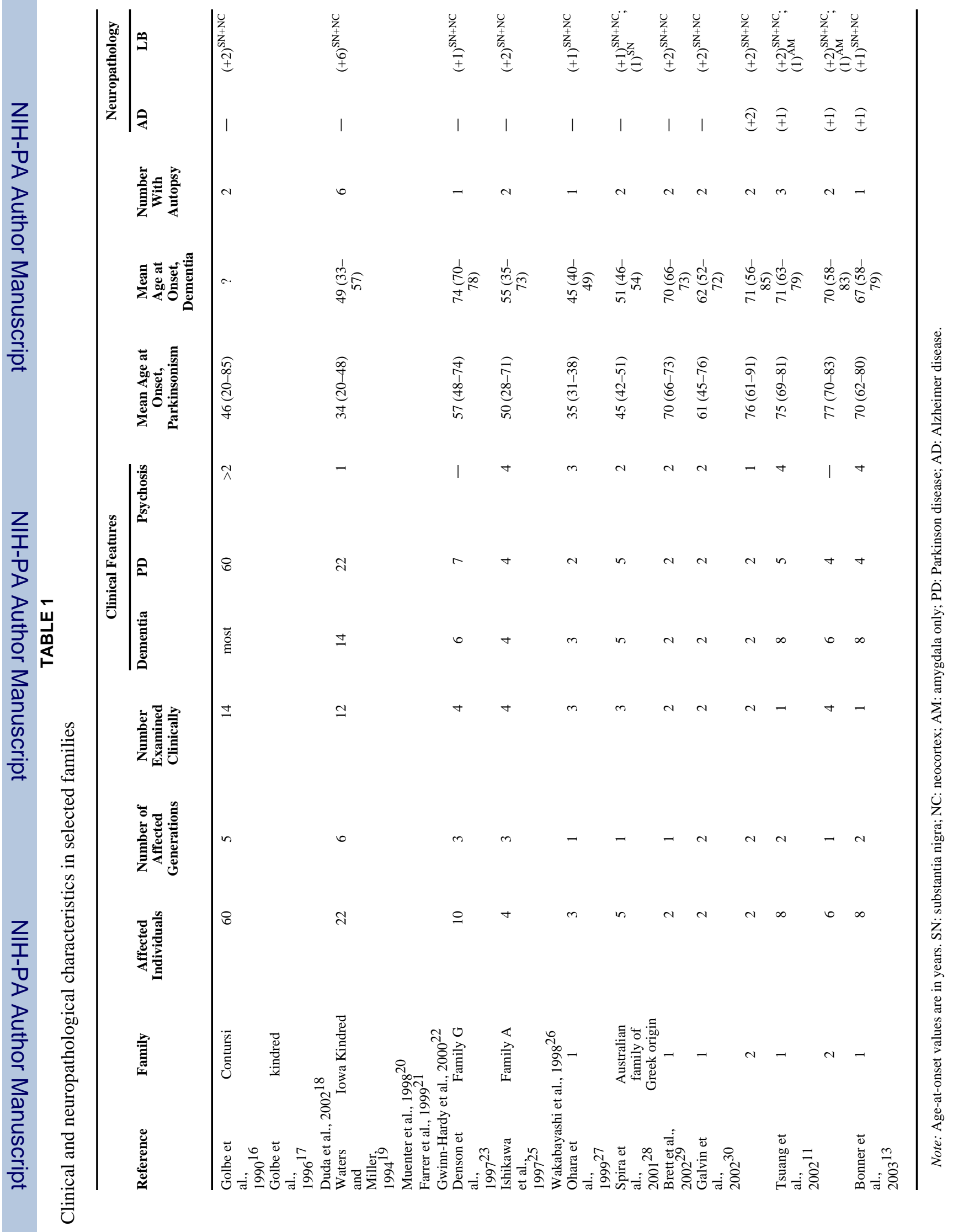

Am J Geriatr Psychiatry. Author manuscript; available in PMC 2006 June 27. 